DOCTRINA

\title{
La naturaleza jurídica de la alta mar en materia de responsabilidad por contaminación
}

\author{
Israel Castro Enríquez (iD \\ Investigador independiente, Ecuador
}

The legal nature of the high seas in pollution liability matters

\begin{abstract}
RESUMEN A la luz del derecho internacional ambiental se estudiaron los regímenes jurídicos, jurisprudenciales y doctrinarios que engloban a la institución de alta mar, ya que tanto la Convención de las Naciones Unidas sobre Derecho de Mar de 1982 como la Declaración de Río sobre Medio Ambiente y Desarrollo de 1992 congregan una desnaturalización a los derechos y obligaciones de protección establecidos en beneficio de alta mar; porque dentro de estos instrumentos internacionales sus efectos y aplicación radican dentro de la teoría soft law, debido al carácter soberano de los Estados y por la escasa técnica legislativa contemplada en su desarrollo normativo; cuestiones que inducen a la formación de vacíos legales que provocan que los casos en materia de contaminación y sobreexplotación de recursos naturales no renovables que yacen en alta mar queden en la impunidad. Por ende, este trabajo invita a la comunidad internacional a modificar la estructura jurídica de la Convención de las Naciones Unidas sobre Derecho de Mar de 1982, en la medida que incorpore una protección efectiva en la Parte VII sobre alta mar; $y$, además incluya en cierto modo al medio ambiente con el objeto de prevenir y reducir potenciales actividades antrópicas que provocan el deterioro del ambiente por los nuevos usos y costumbres instigados en el siglo XXI.
\end{abstract}

PALABRAS CLAVE Alta mar, responsabilidad, medio ambiente, Convención de la Naciones Unidas sobre Derecho de Mar, Declaración de Río sobre Medio Ambiente y Desarrollo.

ABSTRACT In the light of international environmental law, the legal, jurisprudential and doctrinal regimes that encompass the institution of the high seas were studied, since the United Nations Convention on the Law of the Sea of 1982 and the Rio Declaration on Environment and Development of 1992 that both congregates a denaturalization of the rights and obligations of protection established for the benefit of the high seas; because within these international instruments their effects and application lie within the soft law theory, due to the sovereign character of the States and the scarce legislative tech- 
nique contemplated in their normative development; issues that lead to the formation of legal vacuums that cause in cases of pollution and overexploitation of non-renewable natural resources inside the high seas remain in impunity. Therefore, this work invite to the international community to modify the legal structure of the 1982 United Nations Convention on the Law of the Sea, to the extent that it incorporates effective protection in Part VII on the high seas, and includes the environment in some way in order to prevent and reduce potential anthropogenic activities that cause environmental degradation due to the new uses and customs instigated in the 21st century.

KEYWORD High seas, liability, environment, United Nations Convention on the Law of the Sea, Rio Declaration on Environment and Development.

\section{Introducción}

Los usos y costumbres que caracterizan las relaciones del ser humano varían con el paso de los siglos. Esta premisa supone regular el estado de las cosas gracias al afán de satisfacer intereses sociales, políticos y económicos de carácter internacional y nacional. Por ello, los Estados nación, mediante sus esfuerzos de cooperación internacional, instan por legislar la alta mar. Bajo esta premisa se analizará el régimen jurídico de alta mar en dos constantes: ¿Por qué se legisla? ¿Y qué derechos comprende?

Ahora bien, es menester explicar la primera constante que conforma el sistema jurídico de alta mar. Los Estados, al estimar criterios normativos para establecer su legislación, esgrimen tres pilares esenciales: soberanía, territorio y gobierno. La soberanía reviste la voluntad general, al ser un fundamento usado para ejercer una potestad de autoridad o dirección dentro de un territorio. Si bien es cierto la soberanía solo puede ser ejercida en un espacio determinado, por ello se conjuga con el ámbito territorial, que responde al espacio físico explícito dentro del cual la organización estatal se ejerce con potestades intrínsecas. Características jurídicas que recaen en los intereses de un ente estatal, mejor conocido como gobierno; fundamento que lleva a cabo una actividad social, política o económica de una nación a través de la creación de normas jurídicas. Estas concepciones de derecho internacional público revisten un régimen jurídico para establecer una necesidad de control y aprovechamiento en un lugar conocido como res nullius o alta mar, debido a su libertad de navegación y explotación de recursos, los que son de gran utilidad para abastecer los intereses socioeconómicos de cada nación, aunque en ocasiones algunos países no se responsabilizan por ciertas acciones que podrían lesionar los derechos de las personas naturales.

Delimitado el primer punto de debate, es preciso entender qué derechos se atribuyen para el alta mar. Este antecedente obliga a remitirse a los principios que resguardan la alta mar en la Declaración de Río sobre el Medio Ambiente y Desarrollo, normativa que logra atribuirle dos derechos proteccionistas en materia ambiental y 
de desarrollo sustentable, que ipso facto están plasmados en los Principio 8, 11 y $17^{1}$ sobre Prevención, preceptos que guardan concordancia con el Principio 15 referente a Precaución, el mismo que tiene el fin de velar por el medio ambiente e insta a:

los Estados parte la aplicación amplia del criterio de precaución conforme a sus capacidades. Cuando haya peligro de daño grave o definitivo por falta de certeza absoluta, no deberá utilizarse como razón para postergar la adopción de medidas eficaces en función de costos para impedir la degradación del medio ambiente.

Analizadas estas nociones generales de la legislación y los derechos de alta mar, es imprescindible incorporar a la contaminación como una particularidad dentro del régimen jurídico internacional, que debe ser comprendida mediante el mismo ánimo en dos hipótesis. En un sentido genérico, según el Diccionario de lengua española, la contaminación se produce cuando se «altera nocivamente la pureza o las condiciones normales de un medio con agentes químicos o exógenos». Por otro lado, en un sentido jurídico específico, la contaminación debe ser interpretada desde las garantías y derechos otorgados por el derecho internacional ambiental; por ende, vale recalcar que su abstracción siempre debe nutrirse de lo establecido por la investigación científica y los hechos materiales que representa. Con estas presunciones de hecho y derecho, la contaminación es un mal regulado por el derecho partiendo de una teoría científica, que en base a estudios legítimos y eficaces debe ser controlada o reducida a niveles soportables para el bienestar de los seres vivos, en concordancia con las políticas internacionales establecidas en la Declaración de Río, específicamente en el desarrollo sustentable, que garantiza la prevención de un daño eminente en detrimento de vida de los humanos, el ambiente y sus demás organismos.

Justificada la voluntad legislativa en materia de contaminación, la segunda interrogante a interpretar está ligada al sistema de protección establecido para alta mar, presupuesto que esgrime derechos y principios para brindar protección a esta figura en casos de contaminación. En efecto, con la Declaración de Río se promulgan derechos que en realidad se materializan en principios, que deberán cumplirse ipso iure ante la ratificación de este instrumento internacional. Conforme a esta lógica normativa, la protección de alta mar sería eficaz en los Principios 2, 3, 4, 11 y 27 de la Declaración de Río, misma que será analizada y contextualizada en la discusión de este trabajo.

1. Declaración de Río sobre Medio Ambiente y Desarrollo, Río de Janeiro, 14 de junio de 1992. Principio 8: [...] para alcanzar el desarrollo sostenible, los Estados deberán reducir y eliminar las modalidades de producción y consumo insostenible y fomentar políticas demográficas apropiadas. Principio 11: [...] los Estados deberán promulgar leyes eficaces sobre medio ambiente, con objetivos, garantías y prioridades ambientales para inducir una política ambiental. Principio 17: [...] evaluación del impacto ambiental, para cualquier proyecto que pueda ocasionar un impacto negativo en el medio ambiente y la decisión de este sea promulgada por autoridades competentes. 
Algo semejante ocurre con el uso y explotación instigada en los recursos naturales no renovables, estamentos que se analizarán mediante el ejercicio fundado en dos supuestos. El primer postulado presenta dos teorías relativas a: i) el carácter genérico enmarcado por políticas públicas socioeconómicas en beneficio de los intereses estatales, puntualizados en dos tendencias que estiman su concepción legislativa; y, ii) el carácter específico para la promulgación de la norma positiva que incorpora el régimen jurídico aplicable.

El carácter genérico pertenece al consumismo que está parcialmente vinculado con la esfera jurídica de protección para el medio ambiente y la doctrina del desarrollo sustentable promovidos por la Declaración de Río. Tendencia que es accionada de acuerdo con lo dispuesto por las políticas públicas e intereses de cada Estado en materia económica, social y cultural (Serrano Moreno, 2010). Y la segunda eventualidad corresponde a la teoría del uso común, postulado que es establecido para los recursos naturales no renovables y renovables que no están dentro de una jurisdicción o un territorio determinado (Klein, 2005: 318), porque esgrime el régimen jurídico de bien común en beneficio de todas las naciones pertenecientes a la comunidad internacional. Sin embargo, este supuesto involucra constantes polémicas, porque si se tiene en consideración la soberanía ${ }^{2}$ dentro del poder económico y político de los países más desarrollados que tienden a usarlos a favor, provocan el aumento de los niveles de contaminación en el mundo, accionar que en ocasiones no concibe una sanción.

Mientras tanto, la teoría específica unifica valores sociales como procesos legislativos que definen a los recursos naturales como: «todo componente de la naturaleza, susceptible de aprovechamiento por el ser humano para la satisfacción de sus necesidades y que tenga un valor en el mercado» (Vignolo Cueva, 2018: 356), acepción que pregona la teoría científica utilitarista que es discutida por el derecho internacional ambiental. Postulado que al ser incorporado al sistema jurídico de los Estados debe considerar el corolario sociopolítico, porque esta potestad decidirá si se incluyen o no normas en materia de protección para el medio ambiente, que de ser incorporadas garantizarían un amparo directo a los recursos naturales no renovables.

Desarrollado el precepto legislativo, es conveniente analizar el segundo precepto normativo, concerniente a qué derechos regulan los recursos naturales no renovables dentro de la protección vislumbrada para altar mar. Esta prerrogativa tiene dos derechos, uno potencialmente proteccionista para el recurso natural no renovable; y otro radicado en la voluntad soberana estatal para dar uso al recurso natural no renovable.

El primer derecho tiene una concepción proteccionista puramente ambiental que en vinculum iuris guarda concordancia con la política de desarrollo sustentable prescrita en el Principio 23 de la Declaración de Río, que dispone «proteger al medio

2. Figura jurídica que otorga la potestad para usar o disponer libremente el recurso natural no renovable o renovable según las necesidades de cada Estado. 
ambiente y los recursos naturales», axioma que en materia de uso y goce reconoce un amparo de carácter numerus apertus, porque tiene un alcance para alta mar como los recursos naturales no renovables. Sin embargo, el segundo derecho resguarda la voluntad soberana de los Estados para dar uso de los recursos naturales no renovables en el artículo 193 de la Convención de las Naciones Unidas sobre el Derecho de Mar (Convemar) que establece que:

los Estados tienen el derecho soberano de explotar sus recursos naturales con arreglo a su política en materia de medio ambiente y de conformidad con su obligación de proteger y preservar el medio marino.

Supuesto normativo que, al reconocer el uso efectivo del recurso natural no renovable por parte de los países miembro, no vislumbra una protección especial para alta mar, porque solo consagra un reconocimiento para los recursos naturales no renovables; estimación que recae en un vacío normativo que proporciona una libertad para efectuar una sobreexplotación o contaminación a los recursos naturales no renovables dentro de este medio.

Por otro lado, se analizarán: 1) organismos internacionales que promueven la protección ambiental; 2) normativa internacional aplicable; y 3) tribunales de justicia internacional competentes para conocer causas controvertidas. Porque es importante conocer cómo los ordenamientos jurídicos suscitados en derecho internacional público, derecho del mar y derecho internacional ambiental, tienen el ánimo de regular y delimitar concepciones como: alta mar, contaminación y explotación de recursos naturales no renovables dentro de cuerpos normativos específicos y generales.

En efecto, la génesis de los organismos internacionales que promueven la protección ambiental se encuentra regulada por la política internacional; y los objetivos que versan en esta política tienen el fin de precautelar y proteger la vida de los seres humanos. Teoría cimentada bajo presupuestos científicos que convergen en dos factores elementales: la globalización y la sociedad de riesgos.

La globalización dentro del derecho radica en un fenómeno «abstracto en el que intervienen estructuras de distinto carácter, que responden a la libre circulación de factores productivos, tecnología, información y modelos sociales-culturales» (Dreyzin de Klor, 2015: 20-21) de cada nación. Definición que abarca una serie de acciones cotidianas realizadas por el ser humano, las que traen a colación un nuevo ritmo de vida que debe estar regulado mediante la cooperación internacional. Asimismo, la sociedad de riesgos se encuentra premeditada dentro de un fenómeno natural y social que comprende un peligro inminente para el bien jurídico protegido vida. Institución jurídica que en el derecho internacional ambiental responde al «equilibrio ecológico y que principalmente se legisla para establecer un régimen de responsabilidad» (Serrano Moreno, 2010: 176), en materia de daños que es aplicado en supuestos relacionados al uso desmedido de los recursos naturales y contaminación. Hecho 
jurídico que trata de evitar la impunidad de los daños ambientales con el objeto de hacer efectiva su mitigación.

Por consiguiente, al materializar los postulados de globalización y sociedad de riesgos se logra incorporar una política internacional para proteger al medio ambiente. Pero para hacer un llamado a la comunidad internacional, estos preceptos normativos deben ser promulgados por un ente que obligue a las naciones del globo a cumplir ciertos parámetros. Con esta premisa, el único ente de cooperación internacional efectivo para regular y aprobar políticas internacionales a los usos del ser humano del siglo XXI es la Organización de las Naciones Unidas (ONU).

Estimados los apotegmas que plasman los esfuerzos de los Estados para crear una legislación proteccionista con miras al correcto uso de alta mar como un bien común, se puede percibir que existen ciertos vacíos normativos tanto en los principios como la normativa de derecho internacional público y de derecho internacional ambiental que convergen en un sistema jurídico basado en hard law que al final es aplicado como soft law por su falta obligatoriedad en materia de responsabilidad.

Responsabilidad fijada en supuestos de contaminación y sobreexplotación de recursos naturales no renovables en alta mar, ya que su interpretación y empleo genera faltas de sanción que al unísono anulan la mitigación del daño provocado en esta figura marina. Axiomas que determinan un razonamiento jurídico en el cual no existe un control efectivo para garantizar un buen aprovechamiento de bienes comunes para las naciones del mundo y materializan el siguiente problema jurídico: ¿La responsabilidad generada por un caso de contaminación o sobreexplotación en alta mar tienen carácter impositivo de soft law?

Esbozado el planteamiento del problema jurídico, la metodología sistemática será la rectora para el correcto análisis y desarrollo del presente trabajo de investigación. El método pragmático servirá para verificar si la hipótesis planteada es correcta o errónea, mediante la revisión objetiva de los postulados legales, históricos, políticos y sociales en el desarrollo del régimen jurídico internacional de las potestades intrínsecas otorgadas a alta mar.

En este sentido, el marco normativo internacional aplicable para los derechos de alta mar, contaminación y recursos naturales no renovables dentro del derecho internacional ambiental son extensos y vertiginosos; porque existen protocolos y convenciones internacionales que prescriben un régimen jurídico restringido, es decir, no incluyen las tres instituciones integralmente, ya que su desarrollo solo versa en: 1) contaminación y alta mar; ${ }^{3} \mathrm{o}$ 2) contaminación y recursos naturales no

3. Véase el Convenio sobre Prevención de la Contaminación del Mar por Vertimiento de Desechos y Otras Materias, con sus Anexos I, II y III; o el Convenio Internacional sobre Responsabilidad Civil por Daños Causados por la Contaminación de las Aguas del Mar por Hidrocarburos con su Anexo del año 1969. 
renovables. ${ }^{4}$ En vista de esta limitación se selecciona a la Convemar ${ }^{5}$ y a la Declaración de Río $^{6}$ por el hecho de que estos instrumentos internacionales incluyen a todas las categorías de estudio del presente trabajo.

Igualmente, es prioritario considerar el razonamiento y dictámenes de los tribunales internacionales de justicia competentes para conocer causas y establecer una resolución de conflictos pacífica. En este sentido, para el presente trabajo se utilizará el criterio estimado del Tribunal Internacional de Derecho del Mar (Tridemar), ${ }^{7}$ órgano competente institucionalizado por la Convemar con el objeto de dirimir conflictos, otorgar protección efectiva y garantizar los derechos atribuidos para alta mar y las demás figuras de derecho marítimo como de derecho internacional ambiental. ${ }^{8}$

Para mejor proveer, este órgano judicial posee dos jurisdicciones. Una contenciosa, prescrita en el artículo 297 de la Convemar, sujeta a controversias de interpretación o aplicación; y, dentro de la lógica del artículo 298 del mismo cuerpo legal $u t$ supra, se expresan las declaraciones formuladas por los Estados. Y, la jurisdicción consultiva, dispuesta en el artículo 191 de la Convemar, la cual otorga esta capacidad a la Sala de Fondos Marinos para «emitir consultas sobre problemas jurídicos planteados en el orden del día de las actividades de la Autoridad Internacional de Fondos Marinos» (Tridemar, 2016: 9).

\footnotetext{
4. Véase el Protocolo sobre Cooperación, Preparación y Lucha Contra los Sucesos de Contaminación por Sustancias Nocivas y Potencialmente Peligrosas y su Anexo.

5. La Convemar tiene por objeto fomentar una política internacional a favor de ciertas figuras de derecho marítimo y en específico un capítulo dedicado a alta mar, premisa convincente para toda la comunidad internacional. Por ello, este instrumento internacional adoptado en 1982 establece «un régimen exhaustivo dedicado a regular los océanos y mares del mundo en un conjunto de normas jurídicas que rigen usos, recursos y nuevos retos» (OMI, 2020), a fin de preservar y garantizar la paz dentro de procesos judiciales y soluciones de controversias en caso de que lleguen a suscitarse.

6. La Declaración de Río insta a los Estados a dar un efectivo cumplimiento de obligaciones y responsabilidades a través de principios rectores que direccionan el uso y manejo correcto de las políticas de desarrollo sustentable en beneficio del medio ambiente, pero con el objetivo de minimizar y nivelar los efectos del cambio climático.

7. Este órgano fue fundado «en 1994 y atribuyéndosele competencias y facultades con la entrada en vigor del régimen jurídico de la Convemar» (Tridemar, 2016:13). Su jurisdicción radica en el Capítulo XV, Anexo VI, que prescribe Disposiciones Generales, Procedimientos y Competencias.

8. Es menester estimar que la Convemar establece que la competencia del Tridemar es «una jurisdicción obligatoria y dota de la misma atribución a la Sala de Fondos Marinos sobre el incumplimiento de normas ambientales y daños» (Tridemar, 2016: 9), en actividades realizadas en altar mar y otras figuras que comprende el derecho del mar.
} 


\section{Marco teórico y normativo}

Los aspectos generales valen de premisa para fundar la normativa que integra las potestades intrínsecas de alta mar en temas de contaminación y explotación de recursos naturales no renovables. Por lo tanto, esta figura inmersa en el derecho internacional público y en el derecho internacional ambiental se fundamenta en el artículo 87 de la Convemar, al establecer que:

Alta mar está abierta a todos los Estados, sean ribereños o sin litoral. La libertad de alta mar se ejercerá en las condiciones fijadas por esta convención y por las otras normas de derecho internacional. Comprenderá entre otras, para los Estados ribereños y los Estados sin litoral: a) la libertad de navegación; b) la libertad de sobrevuelo; c) la libertad de tender cables y tuberías submarinos; d) la libertad de construir islas artificiales; e) la libertad de pesca; y f) la libertad de investigación científica.

Estas libertades serán ejercidas por todos los Estados teniendo debidamente en cuenta los intereses de los otros Estados en su ejercicio de la libertad de alta mar. ${ }^{9}$

El articulado de la Convemar interpretado ut supra, incluye la libertad de uso para dos tipos de Estados: 1) los Estados ribereños, que poseen acceso al mar sin dificultades geográficas; y 2) los Estados sin litoral, que no poseen acceso al mar por su situación geográfica.

Vale dejar claro que el artículo 87 de la misma Convención no vincula un derecho de protección en materia de responsabilidad en los supuestos de contaminación o explotación de recursos naturales no renovables en alta mar. Pero esta falta de desarrollo jurídico se complementa con la obligación general del artículo 192 que dispone: «Los Estados tienen la obligación de proteger y preservar el medio marino»..$^{10}$ Hipótesis que trata de suplir el vacío de responsabilidad de la Parte VII sobre alta mar, aunque no es suficiente para imponer una sanción efectiva.

Expuesto el alcance normativo de la Convemar en los supuestos de responsabilidad por contaminación o sobreexplotación en recursos naturales no renovables, se evidencia que en el texto no existen otras normas que protejan de manera efectiva a alta mar, en virtud de ello es menester analizar lo expuesto en la Declaración de Río. En efecto este instrumento internacional prescribe en el Principio 7 que:

Todos los Estados deben cooperar con un espíritu de solidaridad mundial para conservar, proteger y garantizar la integridad y salud del ecosistema de la tierra. Porque han contribuido en distinta medida a la degradación del medio ambiente mundial por lo que los Estados tienen responsabilidad común pero diferenciada.

9. Artículo 87, Convención de las Naciones Unidas sobre el Derecho del Mar.

10. Artículo 192, Convención de las Naciones Unidas sobre el Derecho del Mar. 
Con la normativa anterior, corresponde interpretar lo dicho por la jurisprudencia como fuente internacional de derecho. Para ello, es de magna importancia fundar el criterio del Tridemar radicado en la competente Sala de Controversias de Fondos Marinos, dentro del análisis de la Opinión Consultiva ISBA/16/C/13, porque esta resolución dirime y explica el alcance de los derechos ambientales en aras de proteger alta mar y otras figuras de derecho del mar.

En resumen, esta resolución jurisprudencial comprende un estudio sobre conservación y explotación de recursos naturales en el pacífico sur, en el cual se analiza una nueva concepción para los derechos, no solo para los comprendidos de la Zona Económica Exclusiva sino también los otorgados para alta mar. El análisis emprendido fundamenta dos vertientes sobre: 1) responsabilidad de los Estados; y, 2) obligaciones de los Estados, precisando su capacidad para atribuir la responsabilidad de tipo objetiva, que al unísono revoluciona los derechos otorgados a alta mar.

Ergo, la resolución ISBA/16/C/13 analiza este alcance con tres preguntas referentes a:

1) ¿Cuál es la eficacia de la responsabilidad y obligaciones jurídicas en la firma de un convenio o contrato para el desarrollo de actividades de explotación o exploración en la Zona y la relación con el acápite XI de la Convemar? 2) ¿Cuál es el nivel de responsabilidad de un Estado ratificante del incumplimiento prescrito al articulado 153.2 y acápite XI de la Convemar? 3) ¿Qué medidas de mitigación o de reparación debe cumplir el Estado con el fin de acatar las responsabilidades impuestas en el artículo 139 de la Convemar?"1

Estimaciones que serán analizadas a profundidad infra en el apartado sobre fallos internacionales sobre el régimen jurídico de alta mar.

Si bien es cierto los postulados esgrimidos por el Tridemar son de extrema importancia en materia de las potestades intrínsecas de alta mar, no hay que omitir el análisis que vislumbra el criterio de la Corte Interamericana de Derechos Humanos (Corte IDH), dentro de la Opinión Consultiva OC-23/17 interpuesta por la República de Colombia, fallo que exhorta la verdadera posición del Estado frente a las «obligaciones derivadas de los deberes de respetar y garantizar los derechos a la vida y a la integridad personal», ${ }_{12}^{12}$ para la protección al medio ambiente.

Como se afirmó, la Corte IDH de conformidad con el artículo 64.1 de la Convención Interamericana sobre Derechos Humanos, emite su resolución en aras de garan-

11. Véase el Informe Anual del Tribunal Internacional del Derecho del Mar 2011. Resolución, Opinión Consultiva, Tribunal Internacional del Derecho del Mar 2011, ISBA/16/C/13, 1 de febrero de 2011. párr. 49.

12. Véase la Opinión Consultiva sobre Medio Ambiente y Derechos Humanos, OC-23/17, Colombia, Corte Interamericana de Derechos Humanos, Opinión Consultiva, 15 de noviembre de 2017. 
tizar la protección del medio ambiente como un derecho humano a ser arduamente protegido por los Estados. Este análisis pronunciado en la OC-23/17 define cuáles son las obligaciones que los Estados deben cumplir para no violentar el derecho al medio ambiente sano, el mismo que está plasmado en dos axiomas:

1) la interrelación entre los derechos humanos y el medio ambiente; y 2) los derechos humanos afectados por causa de la degradación del medio ambiente, incluyendo el derecho a un medio ambiente sano. ${ }^{13}$

\section{Revisión de la literatura}

\section{Postulados doctrinarios sobre altar mar}

Antes de analizar la posición doctrinaria que congrega la alta mar es conveniente saber su limitación geográfica, bajo esta premisa la Convemar en la Parte VII, Sección 1 de las Disposiciones Generales en el artículo 86 establece:

Alta mar comprende todas las partes del mar no incluidas en la zona económica exclusiva, en el mar territorial o en las aguas interiores de un Estado, ni en las aguas archipielágicas de un Estado archipelágico. ${ }^{14}$

Por ello, la naturaleza jurídica de alta mar está anexada al criterio de autonomía, palabra proveniente del griego antiguo «autos, equivalente al proverbio por sí mismo y nomos, vinculado a ley; que al asociarse originan la facultad de obedecer a sus propias normas» (Valencia Carmona, 2003: 1). En efecto, esta figura jurídica inmersa en la autonomía requiere de un análisis doctrinario dentro del régimen jurídico preestablecido en el derecho internacional público y el derecho del mar.

En consecuencia, alta mar rige en un uso típico y atípico, debido a su falta de soberanía y control en un territorio determinado, doctrina que contraviene el «distintivo del Estado moderno, según el entendimiento convencional, en el cual la soberanía se ejerce exclusivamente sobre un territorio definido» (Cassese, 2005: 7), vicisitud que rompe la teoría general del Estado, pero es completamente válido para entender la naturaleza jurídica de esta figura marítima.

Es así como el derecho marítimo durante los siglos XV al XIX basó su teoría en el principio fundamental de mare liberum, por el deseo de los Estados en su intento de «establecer su soberanía sobre alta mar en aras de monopolizar el comercio y la pesca, pero estos intentos fracasaron a favor del establecimiento del sistema de aguas abiertas» (Klein, 2005: 5). Dentro de esta doctrina, Hugo Grotius afirmó «que las cosas no pueden ser confiscadas o encerradas para convertirse en propiedad» (Klein, 2005: 5).

13. Opinión Consultiva sobre Medio Ambiente y Derechos Humanos, párr. 46.

14. Artículo 86, Convención de las Naciones Unidas sobre el Derecho del Mar. 
Una vez implementado el sistema de aguas abiertas, la alta mar evolucionó jurídicamente en la doctrina del res communis, potestad que radica en la teoría del bien común en beneficio de todos los Estados. En este sentido, este precepto garantiza un trato igualitario y libertad de uso para satisfacer los intereses económicos, sociales y políticas de todas las naciones de la comunidad internacional, siempre y cuando cumpla con el principio del uso compartido.

\section{Postulados doctrinarios sobre los recursos naturales no renovables marítimo}

Este epígrafe se origina en el siglo XIX y a principios del siglo XX, por la llegada de la tecnología y el consumismo. Aunque sus ideales se propagan gracias al interés por el desarrollo económico y político de los Estados, mismos que radican en la explotación excesiva de los recursos naturales no renovables. Problemática que está inmersa en la teoría del uso de bien común en beneficio de la humanidad, doctrina similar a la de alta mar, pero con ciertas especificaciones en materia de 1) ecosistema marino, 2) minería y 3) petróleo.

\section{Ecosistema marino}

Está figura jurídica incorporada en la doctrina de bien común en beneficio de la comunidad internacional involucra un régimen especial, porque si no congregara garantías de prevención y protección generaría repercusiones catastróficas para la vida y el medio ambiente. Por consiguiente, su desarrollo legislativo fue previsto «en 1954, mediante la redacción de un proyecto de convención, en el cual la ONU culminó con la fundación de la Organización Marítima Internacional» (ICPPSO, 2005: 153), organismo que dio apertura y fomentó regulaciones en favor de la protección del ecosistema marino.

Del mismo modo, en 1982 la ONU en la Convemar establece «una serie de deberes generales relacionados con la protección y preservación del medio ambiente marino, así como el tratamiento específico de la contaminación» (Klein, 2005: 147), axiomas que tienen por objeto reducir la explotación insensata e incentivan una garantía para el correcto uso y goce de los mares y océanos.

Sin embargo, esta normativa debe respetar la política internacional y nacional de amparo y preservación del medio marino, vislumbrada por la «cooperación internacional, que es la principal tendencia de regulación sobre la normativa regional, aunque estas dos deben trabajar juntas para otorgar una normativa global» (McConnell y Gold, 2005: 148). Esta iniciativa es apta para la creación de normas jurídicas que contemplen la protección del ecosistema marino y su correcta explotación mediante programas específicos de explotación como mitigación.

Prerrogativas que fomentan y extienden un nivel de protección que engloba «di- 
ferentes estándares que dependen de la fuente o contaminación, instigados por las acciones de un Estado dentro de una zona marina» (Klein, 2005: 148), doctrina que se convierte en una obligación de hacer, bajo el supuesto de respetar el deber objetivo de cuidado y que en caso de negligencia se sancionará a la nación que haya provocado el daño ambiental dentro del ecosistema marino.

\section{Minería}

Este recurso natural no renovable está congregado dentro de la misma teoría de bien común. Según, Tomoko Kake la postura jurídica para otorgarle derechos y atribuciones se fundan con el descubrimiento de los «nódulos minerales que contienen cobre, níquel, cobalto y manganeso ubicados en lecho del océano a tres millas de profundidad» (Kake, 2020: 1-8), acontecimientos que en primera instancia no tenían una regulación y se acogieron a la doctrina de bien común para la humanidad.

Igualmente, esta teoría fundamenta la importancia económica para los «Estados de primer mundo sumado al régimen legal del Nuevo Orden Económico Internacional» (Klein, 2005: 317), en el cual los yacimientos mineros ubicados en el medio marino son un patrimonio común de todos Estados y no son objeto de apropiación para fomentar un uso exclusivo.

La posición ut supra guarda una discusión para su aplicación, porque expone que el régimen jurídico de aplicación para el aprovechamiento de la minería como bien común

no logró establecerse en virtud del principio res communis, el cual permitía a todos los Estados explotar la propiedad común con un respeto razonable de los usuarios, además tampoco se debió considerarlo como res nullius, problema que permitió la adquisición de derechos exclusivos por ocupación o apropiación (Klein, 2005: 318).

Finalmente, la Convemar afirma que la minería en el fondo marino es un bien colectivo para el beneficio de la humanidad. Sin embargo, se instaura «que esta área no es objeto de apropiación o reclamo soberano y que todas las actividades de explotación se regirán por esta convención» (Klein, 2005: 321), pero doctrinariamente seguirá con los postulados sobre res communis incluido el principio de libre mercado, pero respetando las pautas de explotación de mínimos y máximos para evitar posibles daños ambientales.

\section{Petróleo}

Simultáneamente, esta categoría de recurso natural no renovable está adherida a la doctrina del bien colectivo en beneficio de los Estados. Empero, el petróleo dentro de la legislación internacional ambiental tiene un régimen particular de protección, 
debido al grado de contaminación que podría representar para alta mar y el medio ambiente.

Por ello, la comunidad internacional a través del Convenio de Responsabilidad Civil de 1992 regula únicamente «la compensación en el caso de producirse la contaminación de hidrocarburos en los océanos y mares del planeta» (Jacobsson, 2007: 421-422), como consecuencia del daño objetivo incluido en la política de protección al ambiente.

En efecto, la legislación internacional que rige estos incidentes en perjuicio del medio marino en la práctica se «interpreta en base a tres postulados: daños por contaminación, medidas preventivas para minimizar la contaminación y el objeto o causa de la contaminación» (Jacobsson, 2007: 423), fundamentos base para adjudicar si existe o no responsabilidad mediante la teoría del daño objetivo producido por contaminación de petróleo en el medio marino.

Asimismo, esta sanción o reparación para el medio marino no solo debe estar protegida por la fuerza efectiva de un tratado o norma jurídica internacional, sino también por la

cooperación de los Estados al aplicar el derecho internacional existente fundamentado en materia de responsabilidad por daños, anexada a los procesos de solución de controversias dentro de los criterios para dictar una adecuada indemnización (Jacobsson, 2007: 427).

Por último, la doctrina aplicable menciona el criterio de los órganos competentes, los que analizan algunos puntos someros que sirven para determinar la contaminación por petróleo en el medio marino. Los criterios que siempre son implementados para el análisis de la causa responden a tres categorías: pérdida económica pura, daño ambiental y canalización de responsabilidad, que sirven para fundar responsabilidad por contaminación de petróleo, pero no son objeto de estudio en este trabajo de investigación.

\section{Discusión}

\section{Alta mar en la doctrina de res communis}

Si bien es cierto, la alta mar para el derecho internacional público no pertenece a un régimen soberano, porque a priori goza de autonomía. En virtud de este precepto, podemos decir que esta figura jurídica no pertenece a ningún Estado. Pero eso no quiere decir que los Estados no obtengan ciertos beneficios de esta, debido a su particular régimen de bien de uso colectivo.

Por consiguiente, gracias a la interpretación esgrimida por normas internacionales, jurisprudencia y doctrina, la alta mar está fuertemente enlazado con la figura de res communis. Criterio que otorga a esta figura el sentido de bien común o colectivo, 
el que cobra diferentes significados según el régimen legislativo de cada Estado, mismo efecto que difiere en cuanto a su uso u ocupación. En efecto, según Lorenzetti existe una «categoría de bienes que no pertenecen al Estado ni a los particulares para su uso exclusivo» (2010: 7), razonamiento que configura la esencia de la alta mar.

Ergo, la doctrina a partir del res communis converge en un nuevo postulado de protección, mediante la teoría del biocentrismo que esgrime una protección de cumplimiento cuasi estricto en criterios relacionados con el uso común y la sustentabilidad. Enunciados que tienen por objeto garantizar y «evitar el agotamiento o destrucción por el uso masivo del bien colectivo, por lo que estos hechos requieren de una regla limitativa para definir su uso sustentable» (Lorenzetti, 2010: 8), teoría establecida en la normativa de la Convemar y la Declaración de Río.

Empero, el problema que discurre en el carácter de uso colectivo para la alta mar radica en la libertad de uso erga omnes comprendida en un derecho subjetivo de uso, el cual en su sentido material no es excluyente, sino es incluyente. Porque este bien versa sobre un beneficio común, el que debe ser utilizado por todos en sentido amplio (Lorenzetti, 2010: 8). Hipótesis que discierne un alcance sin limitaciones o prohibiciones de goce por una normativa internacional, vacío que permite dar un uso efectivo conforme a los intereses y políticas internas de cada Estado.

En virtud del derecho subjetivo atribuido a esta figura jurídica, no se debería omitir su estatus normativo, ya que su realidad legal «se identifica por su reconocimiento deontológico, en el sentido que su protección debe estar reglamentada» (Alexy, 2004: 9). Postulado doctrinario que sirve para entender la realidad de reconocimiento deontológico de la alta mar, la cual está tipificada en la Convemar, con el fin de evitar la ignorancia de ley, en la hipótesis de la capacidad de uso efectivo para los Estados. Aunque esta tipificación no prevé la contaminación y explotación de recursos naturales no renovables. ${ }^{15}$

Sin embargo, no se debe obviar que la alta mar, al ser un bien común, contiene una legitimación para obrar. Legitimación comprendida por su carácter general y no específico, dado el ánimo de beneficio colectivo. Efecto que garantiza su protección objetiva cuando se vulnerare su esencia o alguna prohibición mandada por ley. Consideración que trae a colación la pregunta sobre quién puede legitimar sus derechos. Esta es respondida con el efecto de res communis, aforismo que «representa el interés colectivo para sus beneficiarios o afectados» (Lorenzetti, 2010: 10). Por esta razón, su legitimación de obrar es amplia y no solo es atribuida al Estado, sino también a entes supraestatales o personas naturales-jurídicas, que se beneficien del bien común o tenga un interés en él.

15. Véase la Convención de las Naciones Unidas sobre Derecho del Mar, Parte VII, Sección I sobre Disposiciones Generales; y la Sección II sobre Conservación y Administración de los Recursos vivos en alta mar. 


\section{Régimen jurídico internacional de alta mar}

La alta mar durante el siglo XX ya poseía un sistema normativo que instauraba un reconocimiento expreso ${ }^{16}$ dado su carácter de bien colectivo supeditado a la doctrina de res communis, aunque no se concebía una protección integral en favor del medio ambiente marino. Asimismo, con el Convenio de Londres de 1972, el Convenio MARPOL de 1973, se implementó un régimen que exigía a los Estados un limitado control marino (Bernie, Boyle y Redgwell, 2009: 386).

Por ello, la comunidad internacional decidió incorporar un nuevo régimen jurídico en la Convemar, y en el artículo 87 es menester interpretar el ánimo del legislador, debido a que se incorporan dos dimensiones de uso y protección para alta mar. La primera dimensión está erradicada en el derecho de libertad que al ser comprendido por su naturaleza jurídica resulta no ser un derecho absoluto, ya que carece de soberanía y, en efecto, se aparta de la esfera del derecho internacional público, aunque se vale del régimen res communis para no ser visto como res nullius y ser susceptible de apropiación. Y su segunda dimensión, vislumbra un derecho de cooperación para fines de investigación científica, categoría jurídica que debe ejecutarse solo con fines pacíficos y mirando el beneficio común para la comunidad internacional.

Vale comentar que el artículo 87 de la Convemar omite el régimen de protección ambiental para alta mar y, en consecuencia, limita tanto los derechos como las garantías ambientales atribuidos por la doctrina del res communis y la cooperación pacífica internacional, que tienen por objeto fomentar el correcto uso y goce de esta figura de derecho de marítimo. ${ }^{17}$ Esto deja como resultado un vacío normativo que permite la perpetración y degradación constante del ambiente por parte de los Estados que se benefician de esta falta de tipificación.

En este sentido, las naciones mediante la Convemar otorgaron garantías mínimas de protección ante las actividades estatales llevadas a cabo en alta mar. No obstante, interpretado el contexto más relevante la responsabilidad expresada como hard law en las categorías de contaminación o sobreexplotación en alta mar se evidencia el carácter de soft law, ya que estos axiomas fueron institucionalizados como opinio iuris equivalentes a derecho consuetudinario (Bernie, Boyle y Redgwell, 2009: 387).

Por otro lado, es conveniente analizar el régimen de protección dilucidado en los principios 8, 11 y 17 en concordancia con el principio 15 de la Declaración de Río. Debido a que estos principios establecen una serie de garantías para el régimen jurídico de alta mar, desde supuestos que verifican cuál es el carácter de efectividad y exigibilidad para los Estados parte. Para ello, este supuesto estudia dos obligaciones

16. Véase la Conferencia de Ginebra sobre el Derecho de Mar de 1958, artículos 24 y 25.

17. Véase la Convención de las Naciones Unidas sobre el Derecho del Mar artículo, Parte XII sobre Protección y Preservación del Medio Marino, Sección II de Cooperación Mundial y Regional. 
fundamentales que podrían o no garantizar la protección de la figura jurídica en discusión.

En efecto, la primera obligación es de dar, puesto que su objeto se manifiesta cuando se ceden derechos en favor o en arreglo de un acto o convenio. Hipótesis que se congrega cuando un Estado parte cede sus intereses nacionales económicos, sociales o políticos con el objetivo de otorgar derechos de protección para alta mar. La segunda obligación es de hacer, que se fundamenta en aras de cumplir con un derecho vislumbrado en un acto o convención, presunción que se cumple cuando el Estado ratificador da fiel cumplimiento y no contraviene los derechos expresados en la convención.

Factores que al interpretarse hacen que esta legislación proprotección ambiental sea híbrida, debido a que su objeto es establecer responsabilidades ante la violación del deber objetivo de cuidado general para el medio ambiente, pero si un Estado no desea ratificar la convención o declaración la hace inexigible para continuar efectuando un daño ambiental. Argumento que $a$ ipso iure e ipso facto plasman el carácter de soft law, aforismo jurídico que deja a los derechos de alta mar en una privación meritoria de un efecto vinculante y coercitivo en materia de responsabilidad o culpabilidad en el caso expreso de violaciones a los recursos naturales no renovables y contaminación al no ser ratificado. ${ }^{18}$

Analizados los principios que más guardan relación para otorgar garantías a alta mar no podemos dejar de lado el régimen de cooperación internacional entre Estados para garantizar la protección eficaz del medio ambiente, establecido en el principio 7 de la Declaración de Río. Axioma que al interpretarse otorga derechos generalísimos y exige la consecución de ciertas obligaciones de protección para las naciones ratificantes.

Los efectos mencionados ut supra versan en potestades típicas, es decir, reposan sobre una norma internacional, corolario que garantiza y exige el cumplimiento de una protección al medio ambiente a los Estados parte. Pero la misma no es mandatoria porque la Declaración de Río al ser un instrumento internacional denominado como declaración tiene un problema de aplicación, principalmente por su carácter potestativo; estructura que desnaturaliza la protección para la contaminación y sobreexplotación de recursos naturales no renovables en alta mar; y, de darse este caso, no existe esta protección por lo que se degrada la conservación eficaz para alta mar.

18. Este efecto jurídico urde cuando en un juicio en el cual estén involucrados derechos pertenecientes a alta mar, se instigue a una falta de aplicación de responsabilidad objetiva directa sobre el daño causado por una persona natural o jurídica perteneciente al sector público o privado, proveniente del incumplimiento de las obligaciones dadas por el convenio internacional, supuesto que elimina una posible reparación integral o medidas de mitigación ambiental. 


\section{Régimen jurídico internacional de la contaminación en alta mar}

Los principios y normativa de protección para el régimen jurídico de alta mar suponen un carácter de hard law, pero por su carácter de exigibilidad expuesta en el apartado desarrollado ut supra recaen en soft law.

Por otro lado, en materia de contaminación del alta mar se congrega un sistema jurídico controvertido porque a partir del Convenio de Londres sobre vertidos de 1972, el Convenio MARPOL de 1973 procedente a contaminación de buques, y otros tratados internacionales relacionados con la institución, no se había incorporado una obligación mandatoria para que los Estados prevean o sean responsables por la contaminación instigada; inclusive aún no se delimitaba un precepto o categoría que regule la contaminación (Bernie, Boyle y Redgwell, 2009: 386), postulado que inducía que los países tengan cierta discreción al momento de instaurar medidas proteccionistas, suceso que ocasionó múltiples impactos y daños a los recursos naturales del planeta.

Es así como esta acepción normativa liberaba a los Estados de responsabilidad en el supuesto que estos contaminen en alta mar, además el contexto del instrumento internacional eliminaba la aplicación del principio de debida diligencia (Bernie, Boyle y Redgwell, 2009: 387), estamentos que instauran un vacío normativo y dejaban en la impunidad los casos relativos a daños al medio ambiente dentro del alta mar.

En consecuencia, a esta evolución normativa la comunidad internacional instauró un régimen jurídico que exige a los Estados el control de las fuentes terrestres de contaminación marina en la Convemar (Bernie, Boyle y Redgwell, 2009: 386).

Al unísono, es importante analizar el contenido del Principio 2 de la Declaración de Río, porque delimita que las naciones poseen «el derecho soberano de aprovechar sus propios recursos según sus propias políticas ambientales y de desarrollo», enfocadas bajo criterios ambientales estimados en políticas públicas para prevenir la contaminación por la explotación de los recursos naturales no renovables en alta mar.

Pero en ningún apartado del Principio 2 se instituye una protección efectiva en aras de dar un correcto aprovechamiento de recursos naturales en alta mar, porque su redacción es específica al expresar el derecho soberano de aprovechamiento de sus propios recursos y no manifiesta el carácter colectivo de protección para dicha figura marítima y, en general, en favor del medio ambiente.

Igualmente, el Principio 3 establece que el derecho al desarrollo sustentable «debe ejercerse de forma equitativa a las necesidades de desarrollo y ambientales», postulado que cobra importancia en armonía del Principio 4, el que justifica que para «alcanzar el desarrollo sostenible, la protección al medio ambiente deberá constituir parte integrante del proceso de desarrollo y no será considerada aislada». En consecuencia, los principios 3 y 4 no otorgan un derecho de protección ambiental a cabalidad, porque este debe equipararse con las políticas internas de desarrollo sustentable 
de cada Estado. Pero no vale obviar que, gracias a este sentido normativo, existe un derecho de protección ambiental enfocado al correcto uso de los bienes.

Aunque, si consideramos las garantías expuestas al tenor literal de los principios analizados, encontramos una protección superficial para los bienes de uso colectivo, debido al carácter técnico del desarrollo sustentable, lógica que está supeditada al criterio soberano de cada nación al ponderar sus intereses socioeconómicos y si los mismos han ratificado o no esta Declaración. Por ello, este plan de protección ambiental será distinto y en ocasiones no cumpliría con las bases generales de la política internacional en materia de medio ambiente por la diversidad jurídica de las naciones, hechos que causan una vez más que las potestades intrínsecas de alta mar puedan violentarse y repercutir en un daño ambiental.

Además, el Principio 11 determina que los países «deberán promulgar leyes sobre el medio ambiente», y el Principio 27 instituye que los Estados «deben cooperar de buena fe para la aplicación de los principios fundados en esta Declaración». Si bien es cierto los principios 11 y 27 son claros y directos, efecto que a ipso iure obligan a los Estados a promulgar leyes que protejan el medio ambiente y que los mismos deberán cooperar de buena fe con lo dispuesto. Acontecimientos que en materia de protección ambiental son gratos, pero carecen de especialidad, debido a que algunos Estados tendrán opiniones heterogéneas para regular diferentes figuras jurídicas. Es así como estos supuestos jurídicos para alta mar perpetran las constantes violaciones a sus derechos conferidos.

En definitiva, las naciones hacen un esfuerzo para proteger al ambiente y la alta mar tal como se establece ut supra, pues la interpretación y analogía son pilares fundamentales para afirmar que un Estado es responsable de un daño al ambiente cuando este viola los presupuestos antes mencionados. Pero teniendo en consideración la normativa dentro de la Declaración de Río, no se esgrime una protección efectiva y materializada en casos de contaminación o daños para la alta mar en ningún articulado; , es aún más grave que en la Convemar no exista un capítulo concreto que proteja la explotación de recursos naturales que confluyen en una potencial contaminación y daños ambientales en alta mar.

\section{Régimen jurídico de los recursos naturales no renovables en alta mar}

Es conveniente dilucidar un vacío normativo en base a la redacción legislativa del artículo 193 de la Convemar, porque si incluimos el criterio de soft law estimado en el Principio 23 de la Declaración de Río que garantiza una protección eficaz para el ambiente y los recursos naturales, los Estados deberían cumplir este postulado normativo al dar uso efectivo de sus recursos.

Por ende, esta interpretación fundamentada en numerus apertus efectiviza una protección para los recursos naturales renovables y no renovables, que en virtud de 
este derecho el Estado que incumpla lo mandado en este principio y dé inicio a una sobreexplotación que provoque un detrimento al medio ambiente, será responsable y sancionado; sin embargo, este criterio estimado en dicho instrumento internacional carece de fuerza y aplicación normativa, en primer lugar por el carácter de soft law de la declaración para efectivizar dicha protección.

No obstante, en el artículo 193 de la Convemar converge una lógica distinta, debido a que expone la capacidad soberana de cada Estado para dar uso de sus derechos de explotación en sus recursos naturales. Categorización que otorga a cualquier nación, si lo considera apto, el derecho de explotar sus recursos naturales no renovables por su propia voluntad soberana, acto que produce una justificación si se contamina al ambiente. Es así como se fundamenta este vacío normativo y cualquier nación podría justificar su actuar con el artículo 193 de la Convemar, debido a que el artículo de la Convención tiene mayor fuerza y vigor por sobre el carácter potestativo de la Declaración.

Por lo tanto, la cooperación y esfuerzos de la comunidad internacional en aras de incorporar derechos ambientales se ven limitados por el estilo legislativo, porque la falta de armonía legislativa entre la Convención y Declaración expresan un vacío normativo que elimina la protección para alta mar y otras figuras de derecho marítimo, exclusivamente por el efecto de fuerza y vigor que se explicó en el párrafo anterior; y como consecuencia de esa interpretación, provoca una explotación desmedida que no tendrá sanción o mitigación alguna si se utiliza el artículo 193 de la Convemar.

\section{Fallos internacionales sobre el régimen jurídico de alta mar}

\section{Opinión Consultiva ISBA/16/C/13 del Tridemar}

Ante la normativa que comprende la alta mar en materia de contaminación y explotación de recursos naturales no renovables, corresponde incorporar el estudio jurisprudencial individualizado en los puntos a resolver de la ISBA/16/C/13, emitida por la Sala de Controversias de Fondos Marinos del Tridemar.

Sin embargo, al conocer la extensión de la resolución ISBA/16/C/13, hay que referirse al razonamiento sobre la responsabilidad y obligaciones de los Estados, en la hipótesis de daño y responsabilidad objetiva, postulados que revolucionan los derechos otorgados a alta mar. Por lo tanto, la ISBA/16/C/13 analiza este alcance con tres preguntas:

La primera pregunta $[. .$.$] expone que los Estados parte poseen dos obligaciones,$ una de gestión y control para que sus contratistas patrocinados cumplan con las salvedades prescritas en la Convención, anexos y demás cláusulas contractuales planteados por cada Estado. La segunda pregunta decreta una obligación directa que está identificada al cumplimiento específico de acciones de prevención impuesta a 
los Estados, muy aparte de la obligación de actuar conforme a las cláusulas contractuales. ${ }^{19}$

Con el análisis de la ISBA/16/C/13 en la primera pregunta se concibe una obligación de hacer, que exige la constante observancia del deber objetivo de cuidado impuesto a los Estados por la Convemar, en concordancia con Principios de la Declaración de Río. Es decir, el Tridemar en esta resolución verifica que las naciones parte deben dar un cumplimiento efectivo de las medidas de prevención y preservación en cualquier hipótesis que presenten actividades de explotación y exploración de recursos naturales no renovables en la zona económica exclusiva o alta mar.

En la segunda pregunta prescribe el grado de responsabilidad de cada Estado según la transgresión cometida, al determinar que «a falta de un efectivo cumplimiento de las debidas obligaciones ratificadas por el Estado o por el incumplimiento del contratista financiado se determinará la responsabilidad», ${ }^{20}$ pero este alcance es más limitado, ya que el incumplimiento debe contener un presagio de causa y de efecto para determinar la responsabilidad del Estado.

En virtud de esta teoría, el presagio causa se define con la falta de diligencia en el deber objetivo de cuidado del Estado; y el presagio efecto debe producir un daño de carácter material irreversible para el medio ambiente. Postulados que al unirse dan la posibilidad de imputar el incumplimiento de la obligación de hacer al Estado que no actuó diligentemente y provocó el daño ambiental.

Finalmente, la tercera pregunta radica en las posibles medidas de reparación o mitigación del daño al ambiente, dispuestas en:

leyes, decretos o reglamentos nacionales o internacionales, que deberán adoptar medidas administrativas, con una doble dimensión: la primera utilizada para garantizar un efectivo cumplimiento de obligaciones del contratista; y la segunda, que no exima de responsabilidad al Estado. ${ }^{21}$

El Tridemar, al referirse a la doble dimensión, insta solo a las obligaciones del Estado y del contratista, mismas que terminan siendo supuestos para efectivizar el plan de manejo ambiental, que tiene por objeto precautelar la mitigación del daño ambiental dentro del tiempo que dure el proyecto de exploración o explotación en el medio marino. Efectos que establecen la responsabilidad de un Estado y la efectiva protección para remediar el daño ambiental, pero nuevamente no incluyen en este razonamiento a la figura de la alta mar, hecho jurídico que deja la salvedad de explotar y contaminar a esta figura sin repercusiones a nivel procesal.

19. Véase el Informe anual del Tribunal Internacional del Derecho del Mar 2011, párr. 55.

20. Véase el Informe anual del Tribunal Internacional del Derecho del Mar 2011, párr. 55.

21. Véase el Informe anual del Tribunal Internacional del Derecho del Mar 2011, párr. 55. 


\section{Opinión Consultiva OC-23/17 de la Corte Interamericano de Derechos Humanos}

La OC-23/17, en su parte pertinente, dicta dos potestades intrínsecas en materia de derechos humanos y de derecho internacional Ambiental. Potestades esgrimidas en: el derecho al medio ambiente sano y el derecho a la vida. Aunque estos dos derechos deben ser entendidos en un mismo sentido, debido a su carácter de protección y respeto por los derechos humanos que están estrechamente ligados a los sujetos del derecho.

Fundamentos clave versados para atribuir responsabilidad y obligaciones a los Estados, que en aras de cumplir con su política interna puedan ocasionar graves daños al medio ambiente. En efecto, gracias a este criterio jurisprudencial de la Corte IDH se analizarán las dos dimensiones desarrolladas ut supra para verificar si alta mar puede ser protegido en base a la presente resolución.

En efecto, el análisis esgrimido de la Corte IDH en la OC-23/17 en un primer plano establece la interrelación entre el medio ambiente y los derechos humanos, discernimiento que estima una protección universal y debe aplicarse solamente ante «la degradación ambiental y los efectos adversos del cambio climático al afectar el goce efectivo de los derechos humanos». ${ }^{22}$

Aunque, lo curioso del razonamiento emitido por la Corte IDH es que presenta una falta de técnica judicial en favor de los derechos del medio ambiente que engloban las potestades intrínsecas de la alta mar. Porque las dimensiones expuestas $u t$ supra solo pueden ser válidas cuando ese derecho de protección al medio ambiente sano está presente en un derecho humano que figure una violación a los derechos de las personas naturales. Efecto que al ser interpretado deja relegados los derechos del medio ambiente y en este caso afectaría a la protección de alta mar.

Incluso dentro de la misma hipótesis, el derecho internacional y los principios de la Declaración de Río exponen que «los Estados reconocieron que los seres humanos constituyen el centro de las preocupaciones relacionadas con el desarrollo sostenible», ${ }^{23}$ criterio que a ipso iure guarda la misma lógica de protección para los intereses de los humanos y no pone un alto o recomendación para el cese de los daños producidos al medio ambiente o en alta mar.

Sin embargo, vale reconocer que la Corte IDH anexa en su razonamiento la resolución del Consejo de Derechos Humanos en cuanto a las responsabilidades ante potenciales daños ambientales, sistema de derecho que garantiza la protección del ambiente dentro del carácter numerus apertus que, sin duda, llega a beneficiar a la alta mar. Es así como establece que las «amenazas ambientales pueden afectar de ma-

22. Opinión Consultiva sobre Medio Ambiente y Derechos Humanos, párr. 47.

23. Opinión Consultiva sobre Medio Ambiente y Derechos Humanos, párr. 52. 
nera directa o indirecta, para el goce efectivo de derechos humanos concretos», ${ }^{24}$ en tres supuestos: contaminación, cambio climático y degradación ambiental.

De manera análoga, nace la relación estrecha entre la protección del medio ambiente en los derechos humanos intrínsecamente en los «sistemas de protección que reconocen la vulnerabilidad de estos por la degradación del medio ambiente». ${ }^{25}$ Vínculo exigido a todo Estado en un efectivo cumplimiento de sus obligaciones de protección y garantía, materializados en derechos sustantivos y adjetivos en favor del ambiente.

En consiguiente, el segundo plano analizado por la Corte IDH versa en cuanto a los derechos humanos afectados por el detrimento al ambiente. Criterio unánimemente fundado sobre el derecho al medio ambiente sano, el cual comprende una connotación colectiva e individual. Para ello, la dimensión colectiva "constituye un interés universal, al proteger otros derechos» ${ }^{26}$ No obstante, su dimensión individual protege toda «vulneración que tenga repercusiones directas o indirectas sobre las personas debido a su conexidad con otros derechos». ${ }^{27}$

Premisas que son exigibles a los Estados mediante cinco obligaciones que otorgan una protección efectiva en favor del derecho al medio ambiente sano, en materia de: «1) garantía a un medio ambiente sano, 2) servicios públicos básicos, 3) protección del medio ambiente, 4) preservación del medio ambiente; y 5) mejoramiento al medio ambiente». ${ }^{28}$

Por ende, estas obligaciones establecen un nuevo derecho autónomo que comprende una protección amplia para el medio ambiente, el que es transmisible para la alta mar. Ergo, este razonamiento logra ampliar la protección del ambiente, aunque por la técnica judicial se trasladan a un segundo plano debido a «su conexidad con una utilidad para el ser humano o por los efectos que su degradación podría causar en otros derechos ${ }^{29}$ intrínsecos otorgados a las personas naturales.

En atención a lo expuesto, se verifica la magnitud del vacío legal que permite en algunos casos la impunidad, la degradación y el menoscabo a los derechos de protección atribuidos a la alta mar y al ambiente en general. Efecto génesis que logra dejar en un segundo plano a las normas del derecho internacional en materia ambiental.

\section{Régimen de responsabilidad aplicado a la alta mar}

En la hipótesis planteada se han desarrollado y estudiado las normas jurídicas y los precedentes internacionales aplicables para garantizar los derechos atribuidos a alta

\footnotetext{
24. Véase, Opinión Consultiva sobre Medio Ambiente y Derechos Humanos, párr. 54.

25. Opinión Consultiva sobre Medio Ambiente y Derechos Humanos, párr. 55.

26. Opinión Consultiva sobre Medio Ambiente y Derechos Humanos, párr. 59.

27. Opinión Consultiva sobre Medio Ambiente y Derechos Humanos, párr. 59.

28. Opinión Consultiva sobre Medio Ambiente y Derechos Humanos, párr. 60.

29. Opinión Consultiva sobre Medio Ambiente y Derechos Humanos, párr. 62.
} 
mar. Pero urge analizar el régimen especial de protección para la alta mar, el que comprende un sistema jurídico de responsabilidad objetiva dentro del criterio de daño ambiental.

En virtud de ello, este apartado tiene su origen en la Convemar, dentro de su parte XI, sección II, artículo 139. Norma prescrita en un marco extenso, con el fin de abarcar la obligación de garantizar el cumplimiento de las disposiciones de la Convemar y la responsabilidad por daños, régimen que establece el primer avance en favor del cumplimiento de derechos proambiente, efecto que a ipso iure comprende integralmente a la alta mar.

No obstante, se debe analizar lo mandado por el artículo 139 y se advierte que para este análisis se utilizará la parte pertinente de dos de sus tres incisos, por lo que este articulado será fraccionado para fines académicos. El primer inciso establece que «los Estados parte estarán obligados a velar por que las actividades en la zona, ya sean realizadas por ellos mismos [...] se efectúen de conformidad con esta parte». Apartado que al ser interpretado no guarda una protección efectiva para la alta mar, porque solo limita la protección de un espacio territorial, referido expresamente a la Zona Económica Exclusiva y al régimen predeterminado de soberanía que gozan los Estados miembro. No obstante, se debe resaltar que esta convención establece una obligación enmarcada en el deber objetivo de cuidado de no dañar al ambiente, precepto normativo que impone una responsabilidad por daños ambientales provocados por contaminación o explotación desmedida de recursos naturales no renovables en la alta mar.

A contrario sensu, el inciso segundo del artículo estimado ut supra en su parte pertinente instituye que «los daños causados por el incumplimiento por un Estado parte o una organización internacional de sus obligaciones con arreglo a esta parte entrañarán responsabilidad», enunciado que otorga una garantía de protección para los derechos proambiente al establecerse de forma amplia, es decir, no es restringida ya que engloba este derecho para cualquier figura marítima.

Aunque este derecho de protección solo versa para la Zona Económica Exclusiva, debido a que el articulado materializa una condición expresa referente al arreglo a esta parte, sentido restrictivo que al final de su interpretación conduce a un vacío legal. Es así como esta falta de técnica legislativa permite continuar con la contaminación o explotación desmedida en alta mar y desvincula esa protección para que la degradación ambiental y los derechos atribuidos para la alta mar sean vulnerados.

Además, se debe traer a colación el contexto del artículo 235 inciso primero de la Convemar en materia de responsabilidad porque dispone: «los Estados son responsables del cumplimiento de sus obligaciones internacionales relativas a la protección y preservación del medio marino». Presupuesto que estima un nivel de responsabilidad limitado, porque sirve para responsabilizar a los países miembro solo en actividades desarrolladas en una jurisdicción específica o en un territorio determinado (Bernie, 
Boyle y Redgwell, 2009: 430), es decir, no se desarrollan in extenso categorías específicas para garantizar una protección efectiva para la alta mar y el medio marino.

Una vez estudiada la normativa relevante en materia de la responsabilidad de los Estados en alta mar, es menester analizar el discernimiento de la jurisprudencia internacional. Criterio escaso, debido a que el Tridemar o las cortes internacionales, como las analizadas ut supra, no han declarado su competencia o interés para resolver un problema que conglomere la responsabilidad por daños objetivos en materia ambiental.

A falta de desarrollo jurisprudencial en materia de responsabilidad objetiva por contaminación y daños al ambiente a cargo del Tridemar, que es el órgano competente instituido por la Convemar, se debe recurrir al caso Prestige o mejor conocido como la Marea Negra, caso insignia en materia de responsabilidad por contaminación que, ante sus controvertidos antecedentes, careció de pronunciamientos internacionales, hecho que obliga a remitirse al criterio de los tribunales nacionales.

Por ello, el caso Prestige sirve para comprender el criterio de soft law dentro del régimen jurídico otorgado en favor de alta mar porque comprende el criterio de responsabilidad, contaminación y recurso natural no renovable, ya analizados en el presente trabajo. Es así como estos elementos componen el régimen de responsabilidad objetiva por daños ambientales provocados por un Estado, persona natural o jurídica, supuestos que forman la hipótesis de incumplimiento de la normativa vigente en la Convemar y los principios evocados en la Declaración de Río.

Para entender mejor la premisa antes expuesta, es primordial referirse a la resolución judicial del Tribunal español en el caso Prestige, en el que se dispuso «que la pretensión de responsabilidad civil por los daños del vertido ha de resolverse conforme a la legislación civil» (Betancor Rodríguez, 2018: 119), dejando de lado el proceso meramente penal que se estaba llevando, puesto que esta causa vinculaba delitos inmersos en obligaciones civiles y existía el ánimo de atribuir una responsabilidad solidaria.

Pero no solo basta con ese argumento, porque el tribunal en su razonamiento dispuso que existía responsabilidad en base cuatro características que corresponden a:

1) Es limitada, por su sistema mixto en el ámbito económico y social; 2) es de carácter objetivo y obligatorio, debido al principio «quien contamina paga»; 3) es exclusiva porque no puede efectuarse ninguna reclamación de indemnización por daños por contaminación marítima por hidrocarburos salvo de acuerdo con los dispuesto en el mismo, y 4) es excluyente porque solo podrá exigirse responsabilidad por esta clase de daños al propietario del buque (Betancor Rodríguez, 2018: 120).

Además, es menester exponer que los axiomas vislumbrados en la sentencia emitida por el tribunal español generaron controversias, como su falta de ejecución debido a graves errores de interpretación en base a criterios civiles y penales atribuidos a cada característica que estaba vinculada con un hecho ilícito que materializaba la res- 
ponsabilidad objetiva por daño ambiental; y los cuestionamientos sobre los hechos y derechos estimados en el caso particular por parte de los tribunales estadounidenses (Betancor Rodríguez, 2018: 120-128).

Por otro lado, el razonamiento del tribunal español es importante en cuanto al daño producido al medio ambiente y su mitigación dentro del caso Prestige, puesto que vincula las instituciones de «1) daño emergente y lucro cesante, 2) daños patrimoniales y ambientales; y 3) daños materiales y morales» (Betancor Rodríguez, 2018: 127) para atribuir responsabilidad objetiva por causal de contaminación, aunque se advierte que estos postulados son realizados exclusivamente para cuantificar y verificar los detrimentos producidos por el siniestro a los municipios y los españoles, dejando al medio ambiente en segundo plano.

$\mathrm{Y}$, a pesar de que reposa en autos las afectaciones producidas al medio ambiente referentes «al litoral costero, playas, superficies rocosas, fondos de la plataforma continental, ecosistemas marinos, aves, biota y sedimentos» (Betancor Rodríguez, 2018: 128), se debe recalcar que el tribunal nunca debate la afectación para alta mar. Dicho esto, el pronunciamiento solo vela por su soberanía y no por el interés colectivo, premisa que al interpretarse evidencia una magna omisión de los derechos otorgados para alta mar y ocasionan que los daños ambientales provocados en dicha figura jurídica eliminen la responsabilidad de los llamados a juicio.

No obstante, se debe reconocer que el tribunal español sí reconoció el daño ambiental sufrido para «los recursos vivos no humanos, la biodiversidad en su conjunto y ecosistemas naturales, sin olvidar los recursos no bióticos que no son objeto de apropiación» (Betancor Rodríguez, 2018: 150). En este análisis se evidencia que el Tribunal reconoce a los recursos no bióticos con carácter de no apropiación, hipótesis que vale de alcance para reconocer el estado de la alta mar, pero nuevamente es relegado al final de todos los reconocimientos in situ.

Finalmente, no hay que omitir el valor de responsabilidad ambiental que este tribunal reconoce, ya que el criterio jurisprudencial español responde «en estricto sentido al derecho de la naturaleza, construida por la condición de patrimonio natural en favor de los ciudadanos» (Betancor Rodríguez, 2018: 151). Razonamiento que es materializado por primera vez dentro del proceso y eleva la protección del medio ambiente a un primer plano, incluso es mirado como daños sufridos a los recursos abióticos, carácter en el que se incluye a la alta mar para otorgarle la protección de sus derechos ambientales enmarcados en la Convemar y la Declaración de Río.

Ergo, gracias a este hecho jurídico relevante se puede validar que la responsabilidad generada en un caso de contaminación o sobreexplotación en alta mar puede tener carácter impositivo de soft law a pesar de que el régimen prescrito en la Convemar sea hard law; ya que en la práctica la carencia de normativa técnica en materia ambiental, y tanto la jurisprudencia internacional como la jurisprudencia nacional en sus razonamientos, dejan en la impunidad los daños ambientales ocasionados en 
la alta mar, debido a que la soberanía de los Estados está por encima de los derechos de bien común y protección efectiva atribuidos para esta institución de derecho marítimo.

\section{Recomendaciones}

En base al análisis presentado en este trabajo, es preciso incorporar una reforma parcial al cuerpo normativo de la Convemar, con el objeto de que este instrumento internacional garantice una protección eficaz para la alta mar y el medio marino dentro de los presupuestos normativos relacionados a contaminación y actividad extractivas que irrespeten los límites permitidos dentro de los recursos naturales no renovables.

En este sentido, se recomienda al legislador internacional incorporar las siguientes categorías jurídicas como garantías en los articulados 87, 139, 193 y 235 de la Convemar, axiomas que dejan al arbitrio de los Estados parte su negociación, firma y entrada en vigor, de acuerdo con la normativa de derecho internacional y preservando el respeto de la soberanía de cada uno de los Estados nación.

Reforma al artículo 87 sobre libertad de la alta mar. Al inciso primero, se incorporará al literal g sobre «la libertad de uso»; categoría que por su contexto deberá congregar un tercer inciso que garantice una protección efectiva relacionada a «alta mar dada su naturaleza jurídica de res communis como bien común en beneficio de la comunidad internacional, la presente Convención le garantiza un régimen de protección especial e incluso obliga a los Estados a preservar y proteger los recursos naturales que se encuentren en su espacio, por ello, se sancionará los daños derivados de contaminación o explotaciones que deterioren el medio ambiente marino».

Reforma al artículo 139 sobre la obligación de garantizar el cumplimiento de las disposiciones de la Convención y responsabilidad por daños. Al texto original del inciso primero del presente artículo se debería incorporar el texto "como para alta mar» después del contexto relacionado a las actividades en la zona, criterio con el que se otorgaría una protección efectiva en el caso que cualquier Estado incumpla la obligación de debida diligencia, precepto normativo que impondrá responsabilidades dentro de los supuestos de contaminación o explotación desmedida de recursos naturales no renovables en alta mar».

Reforma al artículo 193 sobre derecho soberano de los Estados de explotar sus recursos naturales. En el presente articulado se debería agregar un segundo inciso que incluya una protección que tenga el ánimo de evitar y prevenir la libertad de explotación en los recursos naturales no renovables de alta mar, al disponer que: «Los Estados parte con arreglo a su política interna y en aras de fomentar el desarrollo sostenible, están obligados a garantizar el uso correcto y protección para los recursos naturales renovables y no renovables situados en alta mar». 
Reforma al artículo 235 sobre responsabilidad. Finalmente, en este artículo se recomienda incorporar un último inciso aparte del texto integral contenido, el que tendrá por objeto responsabilizar a los Estados dentro de los postulados relacionados a contaminación y actividades extractivas que causen detrimentos en alta mar, para ello se dispondría: «Los Estados parte que hayan contaminado o como producto de actividades extractivas ocasionen un perjuicio grave al medio marino, alta mar y a la comunidad internacional, serán responsables de acuerdo con el derecho internacional debido al incumplimiento de sus obligaciones».

\section{Conclusiones}

La responsabilidad generada en un caso de contaminación o sobreexplotación de recursos naturales no renovables desnaturaliza la protección de las potestades intrínsecas de la alta mar. En virtud de los regímenes jurídicos internacionales, fallos internacionales y responsabilidad por contaminación o explotación desmedida. Postulados que validan en sentido positivo el problema jurídico de este trabajo de investigación.

Es decir, que el carácter material del hard law de la normativa de la Convemar en materia de responsabilidad por contaminación o sobre explotación, tiene carácter de soft law, axioma que igualmente es emblema en la Declaración de Río. Principalmente por las premisas de: 1) soberanía anexada a la potestad de un Estado para no ratificar una convención o declaración que de iure y de facto eliminan la protección de los derechos de alta mar; y 2) técnica legislativa bajo la carencia de especialidad de las normas que protegen derechos de forma parcial, hecho jurídico que concede vacíos o lagunas normativas que limitan la protección y preservación de alta mar.

Sin embargo, este régimen de soft law desaparece parcialmente con la ratificación de una convención o declaración proteccionista de derechos ambientales, porque permite instaurar el hard law, supuesto que garantiza la aplicabilidad del régimen de responsabilidad y permite que estas conductas no queden en la impunidad. Pero esta hipótesis no tendrá mucha efectividad por la falta de técnica legislativa específica, como se demostró en el apartado de las discusiones.

Por otro lado, las limitaciones encontradas en la investigación radican en la polémica actual y novedosa de la hipótesis presentada. Con esta razón los problemas urden en la falta o escasez de legislación, jurisprudencia y doctrina; elementos jurídicos que han sido puntualizados y referidos dentro del análisis del ensayo.

Finalmente, las sugerencias más relevantes versan en dos posturas. La primera vislumbra una reforma para las normas que rigen la figura jurídica de la alta mar en los artículos 87, 139, 193 y 235 de la Convemar, con el objeto de que garanticen la protección y limiten las constantes vulneraciones que podrían suscitarse en arreglo a su falta de técnica legislativa. No obstante, se advierte que el proceso para incorporar las reformas planteadas a la Convemar podría tener dos impedimentos relaciona- 
dos a 1) la efectividad de las negociaciones supeditadas a los intereses soberanos de cada nación; y 2) el carácter costo beneficio que implicaría instalar este proceso en la Asamblea General de las Naciones Unidades.

En efecto la reforma de estos artículos debe ser de tipo parcial, debido a que se debe añadir al texto normativo original para el régimen de responsabilidad dentro de un carácter de numerus clausus. Efecto que garantizará una protección específica para los derechos otorgados en favor de la alta mar en los supuestos contaminación y sobreexplotación de recursos naturales no renovables, pues este hecho jurídico garantizará que estas conductas no queden en total impunidad. Y la segunda enfocada al refuerzo de la cooperación internacional entre Estados para poder dar un austero cumplimiento a lo mandado por la Declaración de Río, con el objeto de paliar el detrimento al medio ambiente y a alta mar.

\section{Referencias}

Alexy, Robert (2004). El concepto y validez del derecho. Barcelona: Gedisa.

DrEYZIN DE KLOR, Adriana (2015). Derecho internacional privado actual. Buenos Aires: Editorial Zavalia.

Betancor Rodríguez, Andrés (2018). Responsabilidad y aseguramiento por daños ambientales. El caso Prestige. Madrid: Boletín Oficial del Estado. Disponible en https://bit.ly/3zıQtOZ.

Bernie, Patricia, Alan Boyle y Catherine Redgwell (2009). International Environmental Law. Nueva York: Oxford University Press.

CAssese, Antonio (2005). International Law in a Divided World. Oxford University Press.

ICPPSO, International Convention for the Prevention of the Pollution of the Sea by Oil (2005). Dispute Settlement in the UN Convention on the Law of the Sea. Cambridge: Cambridge University Press.

Serrano Moreno, José Luis (2010). «La sociedad del riesgo y el derecho de la sociedad». Cuadernos Electrónicos de Filosofía del Derecho (Universidad de Valencia), 21. Disponible en https://bit.ly/3mxvcYI.

McConnell, Moira L. y Edgar Gold (1991). Dispute The Modern Law of the Sea: Framework for the Protection and Preservation of the Marine Environment. Ohio: Case Western Reserve University.

JACOBsson, Måns (2007). «Uniform Application of the International Regime on Liability and Compensation for Oil Pollution Damage». En Tafsir Malick Ndiaye y Rüdiger Wolfrum (eds.), Law of the Sea Environmental Law and Settlement of Disputes Liber Amicorum Judge Thomas A. Mensah. Leiden: Brill.

KLeIn, Natalie (2005). Dispute Settlement in the UN Convention on the Law of the Sea. Cambridge: Cambridge University Press. 
Corte IDH, Corte Interamericana de Derechos Humanos (2017), Opinión Consultiva sobre Medio Ambiente y Derechos Humanos, núm. OC-23/17, Colombia.

OMI, Organización Marítima Internacional (2019). «Convención de las Naciones Unidas sobre Derecho del Mar. Evolución e Historia de la Legislación». Disponible en https://bit.ly/3mKvgIX.

LoRenzetti, Ricardo Luis (2010). Teoría del Derecho Ambiental. Pamplona: Editorial Aranzadi.

Valencia Carmona, Salvador (2003). Derecho, autonomía y educación. México: Instituto de Investigaciones Jurídicas. Disponible en https://bit.ly/3qCfhcv.

Vignolo Cueva, Orlando (2018). «La construcción jurídica de los recursos naturales en Perú». Revista Aragonesa de Administración Pública (Aragón), 17: 351-383. Disponible en https://bit.ly/3 $\mathrm{H}_{6} \mathrm{ag}_{2} \mathrm{~K}$.

KAKE, Tomoko (2020). «Deep-Sea Mining Legislation in Pacific Island Countries: From the Perspective of Public Participation in Approval Procedures». En Quentin Hanich (ed.), Marine Policy. Amsterdam: Elsevier. DOI: 10.1016/j. marpol.2020.103881.

Tridemar, Tribunal Internacional de Derecho de Mar (2016). Responsabilidades y potestades del Tribunal. Hamburgo: Oficina de Prensa del Tridemar.

\section{Agradecimientos}

Expreso un grato agradecimiento a mi mentor Andrés Martínez-Moscoso. En memoria de mi abuela Rosa Amelia Troya Montenegro.

\section{Sobre el autor}

Israel Castro Enríquez es abogado por la Universidad San Francisco de Quito. Actualmente cursa la especialidad en Derecho Ambiental en la Universidad de Buenos Aires. Es Asistente de Investigación en el Instituto de Investigaciones Jurídicas del Colegio de Jurisprudencia de la Universidad San Francisco de Quito. Su correo electrónico es castroisrael31@gmail.com. (D) https://orcid.org/0000-0003-1310-8064. 
La Revista de Derecho Ambiental, del Centro de Derecho Ambiental de la Facultad de Derecho de la Universidad de Chile, es un espacio de exposición y análisis en el plano académico del derecho ambiental. Su contenido se presenta a través de doctrina, jurisprudencia y recensiones, y aborda diversas materias relacionadas con la gestión, institucionalidad y herramientas de protección ambiental y desarrollo sustentable. Se presentan artículos de diferentes autores y autoras en los que se analizan y abordan casos y temas jurídico-ambientales de creciente interés y actualidad.

\author{
DIRECTORA \\ Valentina Durán Medina \\ EDITORES \\ Jorge Ossandón Rosales \\ y Antonio Pulgar Martínez \\ SITIO WEB \\ revistaderechoambiental.uchile.cl \\ CORREO ELECTRÓNICO \\ revistada@derecho.uchile.cl \\ LICENCIA DE ESTE ARTÍ́CULO \\ Creative Commons Atribución Compartir Igual 4.o Internacional
}

La edición de textos, el diseño editorial

y la conversión a formatos electrónicos de este artículo

estuvieron a cargo de Tipográfica

(www.tipografica.io) 